Provided for non-commercial research and education use. Not for reproduction, distribution or commercial use.

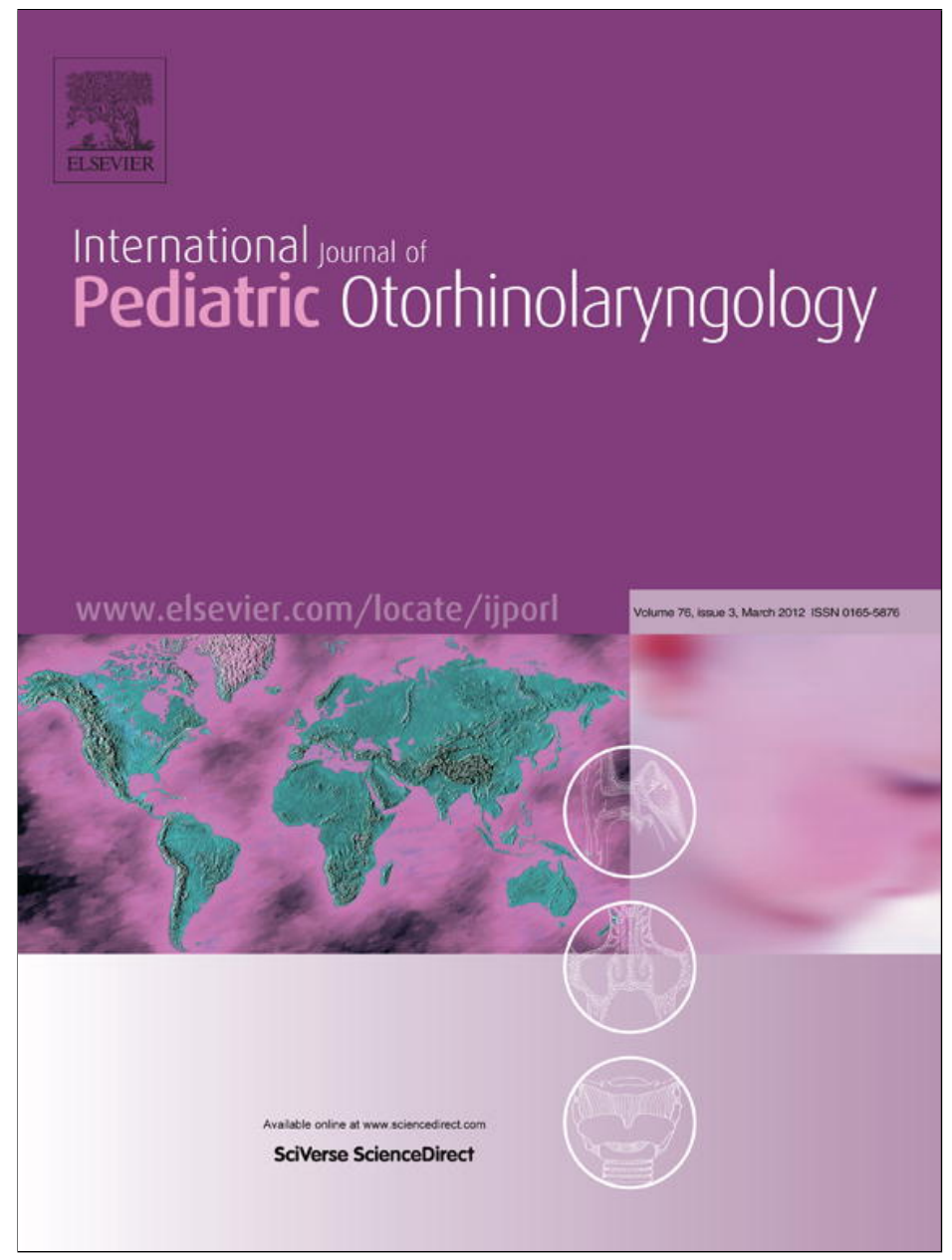

This article appeared in a journal published by Elsevier. The attached copy is furnished to the author for internal non-commercial research and education use, including for instruction at the authors institution and sharing with colleagues.

Other uses, including reproduction and distribution, or selling or licensing copies, or posting to personal, institutional or third party websites are prohibited.

In most cases authors are permitted to post their version of the article (e.g. in Word or Tex form) to their personal website or institutional repository. Authors requiring further information regarding Elsevier's archiving and manuscript policies are encouraged to visit:

http://www.elsevier.com/copyright 


\title{
On the threshold of effective well infant nursery hearing screening in Western Sicily
}

\author{
Francesco Martines $^{\mathrm{a}, *}$, Daniela Bentivegna ${ }^{\mathrm{b}}$, Simona Ciprì ${ }^{\mathrm{b}}$, Claudio Costantino ${ }^{\mathrm{c}}$, Donatella Marchese ${ }^{\mathrm{a}}$, \\ Enrico Martines ${ }^{\mathrm{b}}$ \\ a Università degli Studi di Palermo, Dipartimento di Biomedicina Sperimentale e Neuroscienze Cliniche, (BioNeC), Sezione di Otorinolaringoiatria, Via del Vespro, 129 - 90127 Palermo, \\ Italy \\ ${ }^{\mathrm{b}}$ Università degli Studi di Palermo, Dipartimento di Biopatologia e Biotecnologie Mediche e Forenzi (Di.Bi.Me.F.), Sezione di Audiologia, Via del Vespro, 129 - 90127 Palermo, Italy \\ ${ }^{\mathrm{C}}$ Università degli Studi di Palermo, Dipartimento di Scienze per la Promozione della Salute, Via del Vespro, 129 - 90127 Palermo, Italy
}

\section{A R T I C L E I N F O}

\section{Article history:}

Received 13 November 2011

Received in revised form 22 December 2011

Accepted 26 December 2011

Available online 25 January 2012

\section{Keywords:}

Neonatal screening

Well-infant nursery

Sensorineural hearing loss

Otoacoustic emission

\begin{abstract}
A B S T R A C T
Objective: To determine the feasibility and effectiveness of well-infant nursery hearing screening programme for the early identification of hearing impairment, based on transient evoked otoacoustic emission (TEOAE) with a high "screen sensitivity" reducing the number of more expensive secondary level exams.

Methods: The newborns were screened by non-specialist health workers in well babies nursery at the twentieth day of life for 6 years consecutive. Based on PASS/FAIL criteria and presence/absence of audiological risk factors the newborns were divided into four groups each one with its personal step programme: G1 - PASS without risk factor, free to go home; G2 - PASS with risk factor, retest at the age of 7 months; G3 - FAIL without risk factor, re-screening after 2 weeks for a maximum of four times before audiology assessment; G4 - FAIL with risk factor, retest after 2 weeks.

Results: The coverage rate increased progressively from $89.8 \%$ to $92 \%$. The referral rate was $1.51 \%$ after second stage with a specificity value of $98.78 \%$. The four-stage screening performed for G3 reduced the numbers of global audiology assessment to $0.91 \%$ with a final global specificity of $99.4 \pm 0.4 \%$. Conclusion: Less than $1 \%$ of infants underwent audiological assessment; the false positives resulted $0.62 \%$ with hearing loss global incidence of 2.95/1000 and a mean age of confirmation of 3.5 months of age. It is reasonable to think that this screening programme could be implement to overall 42 Western Sicily birth centres within few years.
\end{abstract}

๑ 2012 Elsevier Ireland Ltd. All rights reserved.

\section{Introduction}

Hearing loss can be considered as the most common congenital disorder in newborns [1]. Early detection and management of hearing loss is crucial for the developmental period for auditory, speech and language acquisition of these neonates. Numerous studies demonstrate that early diagnosis and intervention before six months of age is effective in allowing children with congenital hearing loss to acquire age appropriate cognitive and spoken language skills [2-5]. The potential benefits of early detection can only be realized if an effective newborn hearing-screening programme is performed. In Italy [6], as in other developed countries in the most of birth centres is not present a specialist

\footnotetext{
* Corresponding author at: Via Autonomia Siciliana 70-90143 Palermo, Italy. Tel.: +39091 545666; fax: +390916554271.

E-mail addresses: francescomartines@unipa.it, francescomartines@hotmail.com (F. Martines).
}

audiologist, therefore the neonatal nurse who understands the consequences of undetected hearing loss is an excellent position to emphasize the point. In fact it plays an essential role performing the screening, charting the results and informing the parents about the screening outcomes.

Among the hearing screening methods, the choice of the TEOAEs is a simple, quick, effective, non-invasive method and need using non-specialists without prior audiological experience $[7,8]$. Even if TEOAE tests are generally thought to be easier to administer, their main limit is represented by the referral rate that range from $6 \%$ to $12 \%$ for the first screening [9-11]. According to Regional Health Department in 2003 we started a NHS pilot study on well babies in a country of Western Sicily (Sciacca) using TEOAEs with the purpose of introducing a screen programme with low-cost protocol and need no specialist control, increasing options for successful treatment within six months [12]. Aim project was also to develop an easy and suitable screening programme comfortable with the current objective hearing screening technologies, with high sensitivity (the proportion of 
the children tested with a specified condition, detected by the screening protocol) and specificity (the proportion of the children tested who do not have a specified condition who are correctly identified by the screen protocol) reducing both the number of more expensive secondary level exams and the workload of the only third level speech and hearing centre present in Western Sicily that represent the effectiveness of UNHS recommended by JCIH [2].

\section{Materials and methods}

This study was carried out by the Department of Audiology, University of Palermo, together with the District of Sciacca, screening all the newborns of Sciacca hospital from January 2003 to date [12]. The District of Sciacca was chosen for the screening because in this province there is only a paediatric unit and the percentage of eligible newborns that were screened before hospital discharge or within the first month of life could have been, according to quality benchmarks of $\mathrm{JCIH},>90 \%$. The urban area of Sciacca presents a central population of 40.849 according to 2002 census with approximately $1 \%$ of newborns per year.

The hearing screening was performed with transient evoked otoacoustic emission using the PASS-RETEST criteria [13]. The study protocol was completely explained to parents before discharge and written informed consent was obtained from each patient. Data for each subject were collected using a specific questionnaire answered by the mothers about the presence of: family history of permanent childhood hearing impairment, in utero infections, ototoxic drugs administration, consanguinity. We also considered as perinatal risk factors: cranio-facial abnormality, physical findings such as white forelock, birth weight $<1500 \mathrm{~g}$, slight hyperbilirubinaemia and low Apgar scores [2]. Therefore, according to the presence/absence of the audiologic risk factors, the newborns were divided into two groups: newborn with risk factor and newborn without risk factor. Family Checklists, separate for babies with or without audiological risk factors, have been developed as a type of "roadmap" for parents and providers of newborns, that were also trained on how "Refer" outcomes during the various stages of screening were not to be presented as evidence of hearing loss but rather as indication for further tests to rule out any uncertainty regarding the hearing status of the child; moreover, to not cause undue anxiety, it was decided to test the newborns at twentieth day after birth, corresponding to the second postnatal check without additional cost for 'maternity unit' $[5,14]$. In fact in absence of a specialist screener, and according to data literature we realized that by subjecting newborns to TEOAEs before discharge, within three days of life, there was a high percentage of "Refer" (false positive) also due to moisture, that is the birth debris and retained fluid in the ear canal [15-18].

The instrument used was the ECHOCHECK ${ }^{\mathrm{TM}}$ OAE SCREEANER by Otodynamics, that is based on the nonlinear cross-correlation method (ILO88) of TEOAE recording. The TEOAE screening was conducted by placing a small ILO ECP probe tip from the $\mathrm{ECHOCHECK}^{\mathrm{TM}}$ inside the baby's ear canal; when powered on, the instrument initiated a routine self-calibration before recordings were made. The click rate was approximately 97 per second and each stimulus (at the probe loud-speaker output) consisted of a single $80 \mu \mathrm{s}$ square pulse. To eliminate passive mechanical artefact from the recorded waveform, stimuli were presented in blocks of four stimuli: 3 small positive polarity stimuli followed by one big negative polarity stimulus three times as large. Click peak stimulus level was $84 \pm 3 \mathrm{~dB}$ SPL. Emissions elicited from the outer hair cells in response to the clicks were picked up by the internal microphone of the equipment and were windowed and filtered to remove unwanted signals; all response data outside a window from $4 \mathrm{mS}$ to $10 \mathrm{mS}$, after the stimulus, were removed to eliminate the stimulus signal. The windowed data was then filtered using $24 \mathrm{~dB}$ per octave Butterworth high and low pass filters with corner frequencies of $1.6 \mathrm{kHz}$ and $2.8 \mathrm{kHz}$, respectively. This filtering preserved the 1.5$3.0 \mathrm{kHz}$ octave band, in which most of the useful OAE information is contained. Lower frequencies, which are often contaminated by noise, and higher frequencies, which are sometimes contaminated by "ringing", were attenuated. The test was based on almost 512 responses recorded after the stimulus, corresponding to a maximum time of one minute. The $\mathrm{ECHOCHECK}^{\mathrm{TM}}$ automatically determined the "pass"|"fail" result based on preset binomial statistical probability that an emission has been recorded within the frequency range preserved so the tester/screener could not alter the default settings.

The test was made for three times for each ear, if the first one resulted fail, to value the exam's repeatability, and was performed in a sleeping well-fed neonate; no sedation was required. The instrument was powered by an-inbuilt rechargeable battery that could last up to $10 \mathrm{~h}$ of continuous use. An important quality control feature of this automated instrument was that every test was assigned a unique identification number which could not be altered or selectively deleted by the screener until it was downloaded to the central database after a maximum of 96 tests. Memory capacity includes date and time, test result, ear tested, test ID, ear canal size, OAE and noise intensity data. This feature therefore provided for an independent validation of results recorded by the screeners for each patient.

Based on both PASS/FAIL criteria and presence/absence of prenatal and perinatal audiological risk factors the newborns were divided into four groups: G1 - PASS without risk factor; G2 - PASS with risk factor; G3 - FAIL without risk factor; G4 - FAIL with risk factor.

The study was articulated in more steps depending on group membership; G1: free to go home without advice for the parents; G2: retest at the age of 7 months and/or diagnostic audiology assessment by 24-30 months of age; G3: re-screening after 2 weeks for a maximum of four times (four steps programme) and those who failed were referred for follow-up at the age of 3.5 months to the Tertiary Speech and Hearing Centre; G4: retest after 2 weeks and in case of fail at rescreen the neonate was immediately send to the Tertiary Speech and Hearing Centre.

The role of the Audiology Section of the Palermo University as the only third level speech and hearing centre of Western Sicily was both to assess a global diagnosis by otoacoustic emission (EOAEs) and auditory brainstem responses (ABR) and to programme treatment and rehabilitation of deaf and hearing impaired children.

Statistical analysis was performed using the Matlab ${ }^{\circledR}$ computer program through the means \pm standard deviation (SD) and the standard error (SE).

\section{Results}

The total number of births in the Sciacca hospital during the period 2003-2008 was 3717; of these 3379 full-term newborns were tested as out-patients, with a screening coverage rate of $91 \%$. In particular the newborns born in the hospital of Sciacca in 2003 were 538 and the number of infants whose underwent screening was $483(90 \%)$ with a $10 \%$ (55 neonates) of escaped. In the 2004 the number of newborns was 653 and $90 \%$ of all (585 infants) underwent screening while $10 \%$ (68 neonates) escaped. Of the overall 666 infants born in 2005, the $90.1 \%$ corresponding to 600 newborns were screened. The percentage of screened newborns increased to $92.7 \%$ (547 of the 590 infants) for the 2006. In the 2007,552 infants, corresponding to $91.2 \%$ of the 605 newborns of Sciacca Hospital were screened. In the last year of the pilot study, the percentage of screened was $92 \%$ corresponding to 612 infants of the total of 665 newborns. Therefore the percentage of screened 
Table 1

Four stage hearing screening programme: details.

\begin{tabular}{|c|c|c|c|c|c|}
\hline & \multicolumn{3}{|l|}{ Total } & \multicolumn{2}{|l|}{ Mean \pm SD (SE) } \\
\hline & $\begin{array}{l}\text { FR- } \\
n=3048\end{array}$ & $\begin{array}{l}\text { FR+ } \\
n=331\end{array}$ & $\begin{array}{l}\text { Total } \\
n=3379\end{array}$ & $\begin{array}{l}\text { FR- } \\
n=3048\end{array}$ & $\begin{array}{l}\text { FR+ } \\
n=331\end{array}$ \\
\hline \multicolumn{6}{|l|}{ I Step } \\
\hline Pass & 2949 & 307 & 3256 & $491.5 \pm 54.9(22.4)$ & $51.2 \pm 10.2(4.2)$ \\
\hline Fail & 99 & 24 & 123 & $16.5 \pm 6.6(2.7)$ & $4.0 \pm 2.5(1.0)$ \\
\hline \multicolumn{6}{|l|}{ II Step } \\
\hline Pass & 3008 & 320 & 3328 & $501.3 \pm 52.0(21.2)$ & $53.3 \pm 10.4(4.2)$ \\
\hline Fail & 40 & 11 & 51 & $6.7 \pm 1.9(0.8)$ & $1.8 \pm 1.0(0.4)$ \\
\hline \multicolumn{6}{|l|}{ III Step } \\
\hline Pass & 15 & - & 15 & $2.5 \pm 1.2(0.5)$ & 0 \\
\hline Fail & 25 & - & 25 & $4.2 \pm 2.6(1.0)$ & 0 \\
\hline \multicolumn{6}{|l|}{ IV Step } \\
\hline Pass & 5 & - & 5 & $0.8 \pm 0.7(0.3)$ & 0 \\
\hline Fail & 20 & - & 20 & $3.3 \pm 2.7(1.1)$ & 0 \\
\hline \multicolumn{6}{|c|}{ Audiologic assessment } \\
\hline Pass & 15 & 6 & 21 & $2.5 \pm 1.9(0.8)$ & $1.0 \pm 0.9(0.4)$ \\
\hline Deaf & 5 & 5 & 10 & $0.8 \pm 1.0(0.4)$ & $0.8 \pm 0.7(0.3)$ \\
\hline
\end{tabular}

infants progressively increased from $89.8 \%$ for the year 2003 to $92 \%$ for the last year. A total number of 338 newborns were missed; in particular in 89 cases corresponding to $2.39 \%$ of total newborns, the parents declined screening, while the remaining 249 cases did not come to the appointment ordered at the dismission.

According to presence/absence of audiological risk factor the 3379 screened were divided into two groups: 331 (9.79\%) with audiological risk factor while 3048 corresponding to $90.21 \%$ of the total screened without risk factor (Table 1).

The number of babies referred at the first stage was 123 (3.64\%), 99 of whom belong to group G3 (FAIL without risk factor) while 24 resulted G4 (FAIL with risk factor). After second stage the number of 'FAIL' infants was reduced to 51 with a referred percentage value of $1.51 \%$. Of these 11 infants were with positive audiological risk factor and were sent to the third level speech and hearing centre for a global audiological assessment. In five cases it was diagnosed a prenatal Sensorineural hearing loss (SNHL), unilateral into 2 cases. The percentage value of deafness among well babies with audiological risk factor was $1.51 \%$. The 40 'FAIL' infants belong to G3 underwent a third screening step and, of them 25 babies resulted refer, and were admitted to a fourth screening step. The final number of $\mathrm{G} 3$ babies which underwent to audiological assessment was of 20 and in 5 cases, corresponding to $0.16 \%$ of total well babies without risk factors a bilateral SNHL was diagnosed. The workload of the third level speech and hearing

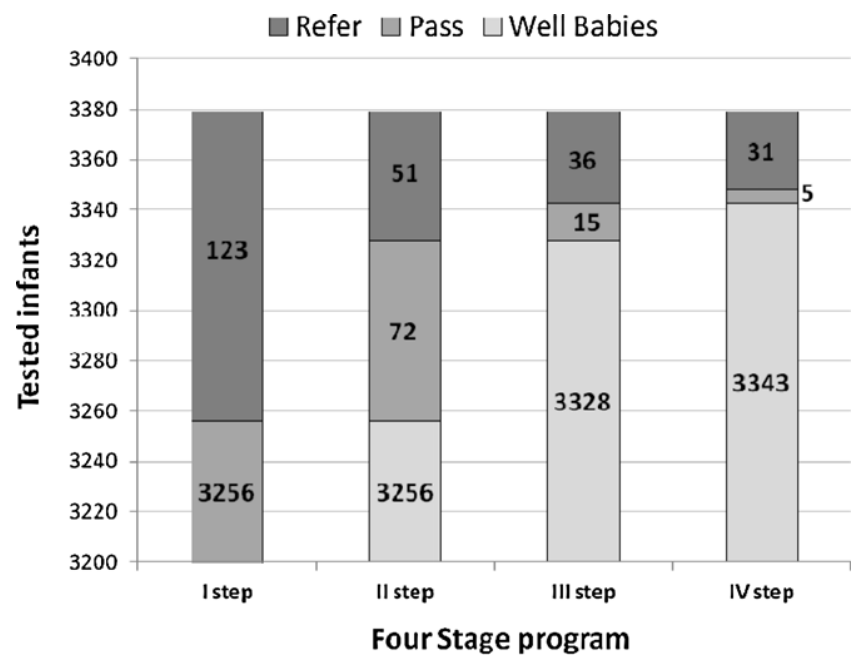

Fig. 1. Details of the infants in the four stage programme. centre was reduced to a total of 31 babies, of whom 21 (0.62\%) resulted false positives (Fig. 1). The global incidence of SNHL, during the period 2003-2008, was estimated to be 2.95/1000 while the incidence of bilateral SNHL resulted 2.36/1000. Overall infants with bilateral deafness had a moderately severe (56-70 dB) hearing loss and began rehabilitation programme before the age of five months.

\section{Discussion}

The goal of newborn auditory screening is to identify infants with significant hearing impairment in the most rapid and costeffective way allowing the opportunity to begin early intervention services for family with infants. Unfortunately, the ideal hearingscreening test for children has yet to be defined and local circumstances may make variations necessary depending on to medical equipment (ABR or/and EOAEs) and, presence/absence of audiological specialist [19-21]. This pilot study carried out in Sciacca hospital from the beginning of 2003, was set out to determine the feasibility and effectiveness of hospital-based UNHS by non-specialists for promoting early detection of congenital and early onset hearing loss in Western Sicily that still now is lacking $[6,12]$.

It is known that the success of the Newborn Screening Programme depends upon the coordinated efforts of many health care professionals; a key role should be represented by neonatal nurse. In fact, to date in Italy in the most of birth centres is not present a specialist audiologist, and the task of implement maternal knowledge and practices regarding audiological risk factors and implications of a misdiagnosed hearing impairment, perform the screening, chart the results and inform the parents about the screening outcomes may led to neonatal nurses [22].

The 'recommended quality benchmarks by the JCIH' regarding the effectiveness of a UNHS focus on the percentage of eligible newborns that must be screened within the first month of life $(\geq 90 \%$ ) [2]. In our pilot study, even if babies underwent screening in the third week of life, after discharge with a consequent higher risk of loss from the screen with respect to those screened before discharge, the coverage rate ranged from $90 \%$ to $92.71 \%$ with a mean value of $91 \%$ in line with JCHI, comparable to the coverage of Mexico, Hong Kong, Brazil and higher than those of $85 \%$ in Malaysia, 67\% in Oman and 45\% in South Africa [14,16,23-26].

The choice of the TEOAE methods is born from the necessity of create an easy screen test that is functional and need using nonspecialists without prior audiological experience [27,28]; moreover they are sensitive to lesion sites common to the well-babies 
Table 2

Specificity values for each stage and relative gain of the experimental protocol.

\begin{tabular}{llllc}
\hline & $\begin{array}{l}\text { Specificity } \\
\text { Step I (\%) }\end{array}$ & $\begin{array}{l}\text { Specificity } \\
\text { Steps I and II (\%) }\end{array}$ & $\begin{array}{l}\text { Specificity } \\
\text { Steps I, II and III (\%) }\end{array}$ & $\begin{array}{l}\text { Specificity } \\
\text { Step I, II, III and IV (\%) }\end{array}$ \\
\hline 2003 & 94.4 & 98.8 & 99.6 & 99.8 \\
2004 & 94.7 & 98.5 & 99.0 & 99.1 \\
2005 & 95.7 & 99.2 & 99.7 & 100 \\
2006 & 97.0 & 98.5 & 99.3 & 99.5 \\
2007 & 97.8 & 98.7 & 98.9 & 99.1 \\
2008 & 98.2 & 99.0 & 99.2 & +5.4 \\
Mean \pm SD (SE) & $96.7 \pm 1.6(0.6)$ & $98.8 \pm 0.3(0.1)$ & $99.2 \pm 0.3(0.1)$ & +4.3 \\
+2.5 & +1.3 \\
+1.0 & $99.4 \pm 0.4(0.2)$ \\
\hline
\end{tabular}

population, namely those of the cochlea. Even if TEOAE tests are generally thought to be easier to administer, their main limit is represented by the referral rate that range from $6 \%$ to $12 \%$ for the first screening while in case of two-step screen, the TEOAE referral rate following the second test could drop to $6-7 \%$. On the contrary AABR tests, that need of specialist control, yield lower firstscreening referral rates (1-4\%) which drop to $1-2 \%$ after the second screening step [9-11].

To reduce false positive rate using TEOAE, remaining in line with the percentage suggested by JCIH ( $\leq 4 \%$ of newborns that failed the screening tests before global audiological assessment), the babies underwent screening at the twenty day of life during the second postnatal check without additional cost for 'maternity unit'; this choose was necessary to eliminate those percentage of refer due to middle ear fluid, negative ear pressure or debris in the ear canals that is more common in the first days of life and could be cause of stress parents. After the first stage screening in fact, our overall referral rate was $3.64 \%$ with a percentage value of false positives of only 3.34\%; therefore we obtained, just with the first step, lower value than the most TEOAE based screening programmes from other countries such as Malaysia (12\%), South Africa (11.1\%), Oman (11\%), Pakistan (10.2\%) and Polonia (4.4\%) maintaining a good coverage percentage [7,14,25,26,29]. The specificity value after first step screening was $96.7 \pm 1.6 \%$ and, this percentage increased to $98.78 \pm 0.3 \%$ after the second stage screening with a total number of 51 referred, 41 of which false positives $(1.21 \%)$ (Table 2).

Usually a hearing screening programme after two referred step orders a global audiological assessment for a refer newborn [30]; the originality of this experimental protocol is that the infants that FAIL without audiological risk factor, below to group G3 and most likely to have a high number of refer, underwent screening 2 weeks later, for a maximum of four times. This measure, without additional cost since it was used the same instrumental, allowed us to lead the total number of newborns admitted to comprehensive audiologic assessment from 51 to 31 newborns with a percentage value of $0.91 \%$ that is more lower than those of $\leq 4 \%$ suggested by JCIH; therefore the number of false positives resulted 21 corresponding to $0.62 \%$ with a final specificity value of $99.4 \pm 0.4 \%$ as showed in Table 2 . Considering that the birth centres of all Western Sicily are forty-two, it is easy to understand how this hearing screening programme articulated with more steps, is able to strongly reduce the number of false positives, with percentage values similar to AABR screening programmes [9-11], and consequently the workload of only tertiary Speech and Hearing Centre of Western Sicily.

A consideration should be made for the infants belongs to group G2 who pass the neonatal screening but have a risk factor; overall these newborns did not require NICU but the possibility of an hearing loss related to neural conduction disorders or "auditory neuropathy/auditory dyssynchrony" cannot be excluded [2]. According to JCHI, even if these disorders typically occur in children who require NICU care, we advised the parents especially for children with prenatal cytomegalovirus infection, to take care to infant's speech-hearing develop and repeating a new test at the age of 7 months. However to date, after eight years from the beginning of this pilot study no false negative was identified.

The mean age of confirmation of hearing loss was of 72 days for the five infants below to G4 which after two failed steps were send immediately to the Tertiary Centre, but at the same time, this procedure gave to the other deafness infants below to G3 (five children), the possibility to undergo an audiologic assessment at the age of 3.5 months as recommended by "principles and guidelines for early hearing detection and intervention programs" of the JCIH (2007) [2]. Moreover all the infants with a diagnosis of SNHL began a rehabilitation programme before the age of 5 months and they have a good speech development and speech intelligibility; this was the main goal of our pilot study because just before this newborn hearing screening programme began the mean age of diagnosed and rehabilitation of hearing loss among infants born in Sciacca hospital was of 7.2 months.

\section{Conclusion}

A major finding in this project was that hospital based universal newborn hearing screening was feasible in the District of Sciacca with active cooperation of the hospital staff, neonatal nurses and caregivers. The study has also demonstrated that TEOAE method performed at the twentieth day of life is suitable for well babies, and that non-specialist staff are capable of screening babies' hearing effectively with minimal training. Moreover the principles of JCHI have been achieved: the infants underwent hearing screening within 1 month of age; all the referred children received an appropriate audiologic and medical evaluations no later than 3.5 months of age; all infants with confirmed permanent hearing loss have received early intervention services before the age of 5 months. According to Sicilian Regional Health Department it is reasonable to think that this screening programme could be implement to overall forty-two Western Sicily birth centres within few years but it should be necessary that all team members will work together to ensure that Western Sicily screening programmes are of high quality and are successful.

\section{Financial disclosure and declaration of interest}

The authors report no conflict of interest. The authors alone are responsible for the content and writing of the paper.

\section{References}

[1] K. Van Naarden, P. Decouflé, K. Caldwell, Prevalence and characteristics of children with serious hearing impairment in metropolitan Atlanta, 1991-1993, Pediatrics 103 (3) (1999) 570-575.

[2] American Academy of Pediatrics, Joint Committee on Infant Hearing. Year 2007 position statement: principles and guidelines for early hearing detection and intervention programs, Pediatrics 120 (4) (2007) 898-921. 
[3] C. Yoshinaga-Itano, D. Coulter, V. Thomson, The Colorado Newborn Hearing Screening Project: effects on speech and language development for children with hearing loss, J. Perinatol. 20 (8 Pt 2) (2000) S132-S137.

[4] M.P. Moeller, Early intervention and language development in children who are deaf and hard of hearing, Pediatrics 106 (3) (2000) E43.

[5] L. Spivak, H. Sokol, Beyond newborn screening: early diagnosis and management of hearing loss in infants, Adv. Neonatal Care 5 (2) (2005) 104-112.

[6] L. Bubbico, G. Tognola, A. Greco, F. Grandori, Universal newborn hearing screening programs in Italy: survey of year 2006, Acta Otolaryngol. 128 (12) (2008) 1329-1336.

[7] W. Szyfter, M. Wróbel, M. Radziszewska-Konopka, J. Szyfter-Harris, M. Karlik, Polish universal neonatal hearing screening program-4-year experience (20032006), Int. J. Pediatr. Otorhinolaryngol. 72 (12) (2008) 1783-1787.

[8] S. Bansal, A. Gupta, A. Nagarkar, Transient evoked otoacoustic emissions in hearing screening programs: protocol for developing countries, Int. J. Pediatr. Otorhinolaryngol. 72 (7) (2008 Jul) 1059-1063.

[9] T. Finitzo, K. Albright, J. O'Neal, The newborn with hearing loss: detection in the nursery, Pediatrics 102 (6) (1998) 1452-1460.

[10] B.R. Vohr, W. Oh, E.J. Stewart, J.D. Bentkover, S. Gabbard, J. Lemons, et al., Comparison of costs and referral rates of 3 universal newborn hearing screening protocols, J. Pediatr. 139 (2) (2001) 238-244.

[11] A.L. Mehl, V. Thomson, The Colorado newborn hearing screening project, 19921999: on the threshold of effective population-based universal newborn hearing screening, Pediatrics 109 (1) (2002) E7.

[12] F. Martines, M. Porrello, M. Ferrara, M. Martines, E. Martines, Newborn hearing screening project using transient evoked otoacoustic emissions: Western Sicily experience, Int. J. Pediatr. Otorhinolaryngol. 71 (1) (2007) 107-112.

[13] S. Griz, G. Mercês, D. Menezes, M.L. Lima, Newborn hearing screening: an outpatient model, Int. J. Pediatr. Otorhinolaryngol. 73 (1) (2009) 1-7.

[14] W. Swanepoel de, R. Hugo, B. Louw, Infant hearing screening at immunization clinics in South Africa, Int. J. Pediatr. Otorhinolaryngol. 70 (7) (2006) 1241-1249.

[15] I. De Souza Jardim, C. Gentile Matas, R. Mota Mamede de Carvallo, Newborn hearing screening with transient evoked otoacoustic emissions and automatic auditory brainstem response, Einstein 6 (3) (2008) 253-261.

[16] M.J. Chapchap, C.M. Segre, Universal newborn hearing screening and transient evoked otoacoustic emission: new concepts in Brazil, Scand. Audiol. Suppl. (53) (2001) 33-36.

[17] B.O. Olusanya, S.L. Wirz, L.M. Luxon, Hospital-based universal newborn hearing screening for early detection of permanent congenital hearing loss in Lagos, Nigeria, Int. J. Pediatr. Otorhinolaryngol. 72 (7) (2008) 991-1001.

[18] C.J. Clemens, S.A. Davis, A.R. Bailey, The false-positive in universal newborn hearing screening, Pediatrics 106 (1) (2000) E7.
[19] K.J. Doyle, B. Burggraaff, S. Fujikawa, J. Kim, Newborn hearing screening by otoacoustic emissions and automated auditory brainstem response, Int. J. Pediatr. Otorhinolaryngol. 41 (2) (1997) 111-119.

[20] J.I. Benito-Orejas, B. Ramírez, D. Morais, A. Almaraz, J.L. Fernández-Calvo, Comparison of two-step transient evoked otoacoustic emissions (TEOAE) and automated auditory brainstem response (AABR) for universal newborn hearing screening programs, Int. J. Pediatr. Otorhinolaryngol. 72 (8) (2008) 1193-1201.

[21] A.L. Berg, B.A. Prieve, Y.C. Serpanos, M.A. Wheaton, Hearing screening in a wellinfant nursery: profile of automated ABR-fail/OAE-pass, Pediatrics 127 (2) (2011) 269-275.

[22] M.P. Moeller, K.R. White, L. Shisler, Primary care physicians' knowledge, attitudes, and practices related to newborn hearing screening, Pediatrics 118 (4) (2006) 1357-1370.

[23] H.M. Yee-Arellano, F. Leal-Garza, K. Pauli-Müller, Universal newborn hearing screening in Mexico: results of the first 2 years, Int. J. Pediatr. Otorhinolaryngol. 70 (11) (2006) 1863-1870.

[24] P.K. Ng, Y. Hui, B.C. Lam, W.H. Goh, C.Y. Yeung, Feasibility of implementing a universal neonatal hearing screening programme using distortion product otoacoustic emission detection at a university hospital in Hong Kong, Hong Kong Med. J. 10 (1) (2004) 6-13.

[25] S.Z. Mukari, K.Y. Tan, A. Abdullah, A pilot project on hospital-based universal newborn hearing screening: lessons learned, Int. J. Pediatr. Otorhinolaryngol. 70 (5) (2006) 843-851.

[26] R. Khandekar, M. Khabori, A. Jaffer Mohammed, R. Gupta, Neonatal screening for hearing impairment-the Oman experience, Int. J. Pediatr. Otorhinolaryngol. 70 (4) (2006) 663-670.

[27] S. Meier, O. Narabayashi, R. Probst, N. Schmuziger, Comparison of currently available devices designed for newborn hearing screening using automated auditory brainstem and/or otoacoustic emission measurements, Int. J. Pediatr. Otorhinolaryngol. 68 (7) (2004) 927-934.

[28] S.G. Korres, D.G. Balatsouras, C. Lyra, D. Kandiloros, E. Ferekidis, A comparison of automated auditory brainstem responses and transiently evoked otoacoustic emissions for universal newborn hearing screening, Med. Sci. Monit. 12 (6) (2006) CR260-CR263.

[29] L. Ali, S. Siddiq, M.A. Khan, S. Maqbool, A hospital-based universal newborn hearing screening programme using transient evoked otoacoustic emission (TEOAE), Pakistan Pediatr. J. 24 (2000) 117-125.

[30] J.L. Johnson, K.R. White, J.E. Widen, J.S. Gravel, M. James, T. Kennalley, et al., A multicenter evaluation of how many infants with permanent hearing loss pass a two-stage otoacoustic emissions/automated auditory brainstem response newborn hearing screening protocol, Pediatrics 116 (3) (2005) 663-672. 